\title{
Recursion Formulae for Hypergeometric Functions
}

\author{
By Jet Wimp
}

I. Notation. The series definition for the generalized hypergeometric function is

$$
{ }_{P} F_{Q}\left(\begin{array}{l}
a_{P} \\
b_{Q}
\end{array} \mid x\right)=\sum_{k=0}^{\infty} \frac{\left(a_{P}\right)_{k} x^{k}}{\left(b_{Q}\right)_{k} k !}
$$

where

$$
(\alpha)_{k}=\Gamma(\alpha+k) / \Gamma(\alpha)
$$

is Pochhammer's symbol and the shorthand product notation above will be used throughout this paper. In general, where a parameter has a subscript which is a capital letter, the repeated product notation is understood:

$$
\left(a_{P}\right)_{k}=\prod_{j=1}^{P}\left(a_{j}\right)_{k}, \quad\left(n+b_{Q}\right)=\prod_{j=1}^{Q}\left(n+b_{j}\right), \quad \text { etc. },
$$

and the ${ }^{*}$ notation

$$
\left(1+b_{Q}-b_{h}\right)^{*}=\prod_{j=1 ; j \neq h}^{Q}\left(1+b_{j}-b_{h}\right)
$$

indicates the term corresponding to $j=h$ is to be deleted.

If one of the $a_{i}=0$ or a negative integer, then (1) always converges, since it terminates. Otherwise it converges for all finite $x$ if $P \leqq Q$ and for $|x|<1$ if $P=$ $Q+1$. In this case, however, the function can be analytically continued into the cut plane $|\arg (1-x)|<\pi$, and we shall often denote by ${ }_{Q+1} F_{Q}(x)$ not only the series (1), whenever it converges, but also the analytic continuation of the series. If $P>Q$ +1 , the series does not converge (unless it terminates) and if one of the $b_{j}$ is 0 or a negative integer, the series is not defined. If one of the $a_{i}$ equals one of the $b_{j},{ }_{P} F_{Q}(x)$ reduces to $P_{-1} F_{Q-1}(x)$ and such a case is always excluded from consideration in this paper. We assume all ${ }_{P} F_{Q}$ 's are irreducible.

Equation (1) can be given an interpretation for $P>Q+1$ by means of the $G$-function

$$
\frac{\Gamma\left(b_{Q}\right)}{\Gamma\left(a_{P}\right)} G_{P, Q+1}^{1, P}\left(\begin{array}{l|l}
-x & \begin{array}{l}
1-a_{P} \\
0,1-b_{Q}
\end{array}
\end{array}\right)
$$

and (5) is (1) (or its analytic continuation) if $P \leqq Q+1$. The $G$-function can be defined by a Mellin-Barnes contour integral.

For a treatment of the generalized hypergeometric function and the $G$-function, see [1].

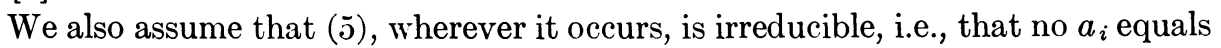
any $b_{j}, i=1,2, \cdots, P, j=1,2, \cdots, Q$.

Received August 28, 1967. 
II. Introduction. The subject of the recursion relations satisfied by hypergeometric functions occupies a prominent place in the literature of special functions. The functions of this type for which recursion formulae have been given are usually special cases of the functions

$$
U_{n}(\lambda)=\frac{\left(a_{P}\right)_{n} \lambda^{n}}{\left(b_{Q}\right)_{n}(\gamma+n)_{n}}{ }_{P+1} F_{Q+1}\left(\begin{array}{c}
n+a_{P+1} \\
n+b_{Q}, 2 n+\gamma+1
\end{array} \mid \lambda\right),
$$

or of the polynomials

$$
P_{n}(z)=\frac{1}{\Gamma\left(d_{T}\right)}{ }_{R+2} F_{T}\left(\begin{array}{c}
-n, n+\gamma, c_{R} \mid z \\
d_{T}
\end{array}\right),
$$

or

$$
Q_{n}(z)=\frac{1}{\Gamma\left(d_{T}\right)}{ }_{R+1} F_{T}\left(\begin{array}{c|c}
-n, c_{R} \\
d_{T}
\end{array} \mid z\right) .
$$

It can be shown that (6)-(8) obey linear recursion relationships of the form

$$
\sum_{\nu=0}^{p}\left[k_{\nu}+x l_{\nu}\right] \Phi_{n+\nu}=0,
$$

where $x=1 / \lambda$ for (6), $x=z$ for (7) and (8), and $k_{\nu}=k_{\nu}(n), l_{\nu}=l_{\nu}(n)$ depend on the particular function, but not on $z$ or $\lambda$. Also, $k_{0}=1, l_{0}=0$, and $\rho$ depends on the number of numerator and denominator parameters in the hypergeometric function: $\rho=\max [P+1, Q+2]$ for $(6), \rho=\max [T+1, R+2]$ for $(7)$ and $(8)$.

$U_{n}(\lambda)$ can be given an interpretation for $P>Q+1$ by means of the $G$-function

$$
\begin{aligned}
& U_{n}(\lambda)=\frac{\Gamma\left(b_{Q}\right)}{\Gamma\left(a_{P}\right)}(-)^{n} \tau_{n} G_{P+1, Q+2}^{1, P+1}\left(\begin{array}{c|c}
1-a_{P+1} \\
-\lambda \mid \begin{array}{c}
1 \\
n-\gamma, 1-b_{Q}
\end{array}
\end{array}\right), \\
& \tau_{n}=(2 n+\gamma) \Gamma(n+\gamma) / \Gamma(n+\beta+1),
\end{aligned}
$$

provided $a_{i}$ is not 0 or a negative integer, $i=1,2, \cdots, P+1$.

There exists a duality between the functions (7) and (10). For instance, we have, under a variety of conditions (see [2, Eq. (2.6)] and also related expansions in [3], [4]),

$$
\begin{aligned}
& G_{Q+T+1, P+R+1}^{P+R+1,1} \\
&\left(-\frac{1}{\lambda z} \mid \begin{array}{c}
1, b_{Q}, d_{T} \\
c_{R}, a_{P+1}
\end{array}\right)= \frac{\Gamma\left(c_{R}\right) \Gamma\left(a_{P}\right)}{\Gamma\left(b_{Q}\right)} \sum_{n=0}^{\infty}(-)^{n}(n+1)_{\beta} \\
& \times U_{n}(\lambda) P_{n}(z)
\end{aligned}
$$

and if, in this multiplication formula, $z$ is replaced by $z / \gamma$ and $\gamma \rightarrow \infty$, a similar expansion in terms of $Q_{n}(z)$ results.

In fact, any function analytic at $z=0$ can be expanded in a series of the polynomials $P_{n}$ or $Q_{n}$, and Fields and Wimp studied such expansions from the standpoint of basic series in [6]. Linear combinations of $P_{n}, Q_{n}$ also occur in classes of rational approximations to generalized hypergeometric functions, see [7] and the references given there.

For $R=0, T=1, P_{n}$ is related to the Jacobi polynomial, as we have seen, and $Q_{n}$ to the Laguerre polynomial. Here $\rho=2$, and the recurrence formulae are classical. For $R=0, T=0, P_{n}$ is the Bessel polynomial, whose recursion formula and other properties have recently been studied by a number of writers, see [8]. 
Recursion formulae for $P_{n}$ for $R=1, T=2(\rho=3)$ have been studied for various special values of the parameters, see [9]. For values of $\rho>3$, i.e., larger values of $R$, $T$, no general results seem to exist in the literature, although general formulae for $\rho=3$ have been derived but not published, [6].

When $P=1, Q=0$, then $\rho=2$ and $U_{n}(\lambda)$ is related to the Jacobi function, $Q_{\boldsymbol{n}}{ }^{(\alpha, \beta)}$, whose recursion formula is given in [5]. No general formulae for larger values of $P, Q$ seem to be known. However, for special values of $\gamma$ and $\beta$, the recursion formula for $P=2, Q=0$ is given in [3], where it was also shown that $U_{n}(\lambda)$ could be computed by using (9) in the backward direction.

Since $U_{n}(\lambda)$ can often be computed by using (9) in the backward direction, and $P_{n}$ and $Q_{n}$ always by using (9) in the forward direction, it is quite desirable to have closed form expressions for $l_{\nu}, k_{\nu}$. It was previously doubted that such expressions existed, since the derivation of particular recursion formulae has hithertofore involved solving systems of algebraic equations whose complexity increases rapidly with $P, Q, R$ and $T$.

In this paper, we determine closed form expressions for the coefficients in the recursion formula for $U_{n}(\lambda)$. These coefficients are terminating hypergeometric functions of unit argument. We show that $U_{n}(\lambda)$ satisfies one and only one recursion relation of type (9) of a certain order and none of a lower order. We next find a number of other solutions of (9), considered as a difference equation. It turns out that certain of these solutions are closely related to $P_{n}$, and by specialization of a certain parameter, we are able to determine the recursion formula for $P_{n}(z)$. Next, by taking a limit as $\gamma \rightarrow \infty$, we find the recursion formula for $Q_{n}(z)$.

The author is grateful to his colleagues, Yudell Luke and Jerry Fields, for a number of helpful comments and suggestions.

\section{Results.}

Theorem 1. Let $P, Q, n$ be integers $\geqq 0$. Let $\beta, \gamma, a_{i}, b_{j}, i=1,2, \cdots, P, j=1$, $2, \cdots, Q$ be complex constants such that none of the quantities $\beta+1, a_{i}, b_{j}, \gamma$ are negative integers or zero. Let $\lambda$ be a complex variable, finite and $\neq 0$, and let $a_{i}=\beta+1$ for $i=P+1$. Then the following statements are true:

(1) the functions $U_{n}(\lambda)$ as given by (10) satisfy the difference equation

$$
\sum_{\nu=0}^{\sigma}\left[A_{\nu}+\frac{B_{\nu}}{\lambda}\right]_{\Phi_{n+\nu}}(\lambda)=0, \quad \sigma=\max [P+1, Q+2],
$$

where

$$
\begin{aligned}
A_{\nu}= & \frac{(-)^{\nu}(2 n+\gamma)_{\nu}}{\nu !(n+\gamma)_{\nu}}(n+\beta+1) \\
& \times{ }_{\nu P+3} F_{P+2}\left(\begin{array}{c}
-\nu, 2 n+\gamma+\nu, n+a_{P+1}+1 \\
2 n+\gamma+\sigma+1, n+a_{P+1}
\end{array} \mid 1\right), \\
B_{\nu}= & \frac{(-)^{\nu}(2 n+\gamma)_{\nu+1}(n+\beta+1)_{\nu}\left(n+b_{Q}\right)}{\Gamma(\nu)(n+\gamma)_{\nu}\left(n+a_{P+1}\right)} \\
& \times_{Q+2} F_{Q+1}\left(\begin{array}{c}
1-\nu, 2 n+\gamma+\nu+1, n+b_{Q}+1 \\
2 n+\gamma+\sigma+1, n+b_{Q}
\end{array}\right)
\end{aligned}
$$

$\left(A_{0}=1, B_{0}=B_{\sigma}=0\right)$; 
(2) other solutions of (12) are

Case A. $\sigma=Q+2 ; p<Q+1$ or $P=Q+1,|\arg (1-\lambda)|<\pi ;$ (here $U_{n}(\lambda)$ is given by (6));

$$
\begin{aligned}
\psi_{n}(\lambda)= & \frac{(-)^{n(P+1)} \tau_{n} \lambda^{-n}}{\Gamma\left(b_{Q}-n-\gamma\right) \Gamma\left(n+\gamma+1-a_{P+1}\right) \Gamma(1-\gamma-2 n)} \\
& \times{ }_{P+1} F_{Q+1}\left(\begin{array}{c}
a_{P+1}-n-\gamma \\
b_{Q}-n-\gamma, 1-\gamma-2 n
\end{array} \mid \lambda\right), \\
\phi_{n}^{[h]}(\lambda)= & \frac{\tau_{n}}{\Gamma\left(2-b_{h}-n\right) \Gamma\left(n+\gamma+2-b_{h}\right) \Gamma\left(1+b_{Q}-b_{h}\right)} \\
& \times{ }_{P+1} F_{Q+1}\left(\begin{array}{c}
1+a_{P+1}-b_{h} \\
\left(1+b_{Q}-b_{h}\right)^{*}, 2-b_{h}-n, n+\gamma+2-b_{h}
\end{array} \mid \lambda\right),
\end{aligned}
$$

$h=1,2, \cdots, Q$

Case B. $\sigma=P+1 ; P>Q+1$ or $P=Q+1,|\arg (1-1 / \lambda)|<\pi$;

$$
\begin{aligned}
\theta_{n}{ }^{[h]}(\lambda)= & \frac{\tau_{n}\left(a_{h}\right)_{n}(-)^{n}}{\Gamma\left(n+\gamma+1-a_{h}\right) \Gamma\left(1+a_{h}-a_{P+1}\right)} \\
& \times{ }_{Q+2} F_{P}\left(\begin{array}{c}
\left.n+a_{h},-n-\gamma+a_{h}, 1-b_{Q}+a_{h} \mid \frac{(-)^{Q+P+1}}{\lambda}\right), \\
\left(1+a_{h}-a_{P+1}\right)^{*}
\end{array}\right.
\end{aligned}
$$

$h=1,2, \cdots, P+1$

(3) none of the functions above satisfy any other difference equation of type (12), with $A_{0}=1, B_{0}=B_{\sigma}=0$, of order $\leqq \sigma$.

Note. We assume $U_{n}$ is not reducible for all $n$, i.e., no $b_{i}$ equals any $a_{j}$ or $\beta+1$. However, for particular values of $n, U_{n}$ may be reducible. Such will be the case if any $a_{j}=r+\gamma+1, j=1,2, \cdots, P+1, r$ an integer $\geqq 0$.

Proof. First we note that

$$
{ }_{M+2} F_{M+1}\left(\begin{array}{c|c}
-\nu, \nu+\mu, 1+a_{M} \\
\mu+r, a_{M}
\end{array} \mid 1\right)=0, \quad \nu, r=0,1,2, \cdots,
$$

for $M<r \leqq \nu$, as can be seen by writing out the $\nu$ th difference with respect to $x$ of $\prod_{t=1}^{\nu-r}(x+r+\mu-1+t) \prod_{j-1}^{M}\left(x+a_{j}\right)$ at $x=0$. This shows that, if (13) and (14) are true, then $A_{\nu}=0, \nu>\sigma$ and $B_{\nu}=0, \nu \geqq \sigma$, in particular, that $B_{\sigma}=0$, as stated.

Next, we remark that if $P<Q+1$, or $P=Q+1$ and $|\arg (1-\lambda)|<\pi$, then $U_{n}(\lambda)$ is precisely (6). If $P>Q+1$ or $P=Q+1$ and $|\arg (1-1 / \lambda)|<\pi$, then $U_{n}(\lambda)$ is a sum of the functions $\theta_{n}{ }^{[h]}(\lambda), h=1,2, \cdots, P+1$. See [10].

Let $P<Q+1$ or $P=Q+1$ and $|\lambda|<1$. By substituting $U_{n}(\lambda)$ into the difference equation and equating to zero the coefficient of $\lambda^{n+k}$, we find that the theorem demands that

$$
S_{1}(k)+S_{2}(k) \equiv 0,
$$

where

$$
\begin{aligned}
& S_{1}(k)=\left(n+b_{Q}+k\right) \sum_{\nu=0}^{\sigma} \frac{\tau_{n+\nu} A_{\nu}}{\Gamma(k-\nu+1) \Gamma(2 n+\nu+k+\gamma+1)}, \\
& S_{2}(k)=\left(n+a_{P+1}+k\right) \sum_{\nu=1}^{\sigma-1} \frac{\tau_{n+\nu} B_{\nu}}{\Gamma(k-\nu+2) \Gamma(2 n+\nu+k+\gamma+2)} .
\end{aligned}
$$


Now substitute the functions $\phi_{n}{ }^{[h]}$ into (12) and equate to zero the coefficient of $\lambda^{k}$. The result is

$$
S_{1}\left(k+1-n-b_{h}\right)+S_{2}\left(k+1-n-b_{h}\right) \equiv 0, \quad h=1,2, \cdots, Q,
$$

with the same value of $\sigma$ as above.

Substituting $\psi_{n}(\lambda)$ into (12) and equating to zero the coefficient of $\lambda^{-n+k}$, we see we must have

$$
S_{1}(k-2 n-\gamma)+S_{2}(k-2 n-\gamma) \equiv 0 .
$$

Finally, let $P>Q+1$ or $P=Q+1$ and $|\lambda|>1$ and consider the functions $\theta_{n}{ }^{[h]}(\lambda)$. Proceeding as above, we see that we must have

If (19) is multiplied by $\Gamma(k+1) \Gamma(2 n+\sigma+k+\gamma+1)$ which is defined for all $k$ in some right half-plane, then (19) becomes a polynomial in $k$, and we see that a necessary and sufficient condition for (19) to hold is that

$$
\begin{gathered}
\left(n+b_{Q}+k\right) f_{1}(k)+\left(n+a_{P+1}+k\right) f_{2}(k) \equiv 0, \\
f_{1}(k)=\sum_{\nu=0}^{\sigma}(-)^{\nu}(-k)_{\nu}(2 n+k+\nu+\gamma+1)_{\sigma-\nu} \bar{A}_{\nu}, \\
f_{2}(k)=\sum_{\nu=1}^{\sigma-1}(-)^{\nu-1}(-k)_{\nu-1}(2 n+k+\nu+\gamma+2)_{\sigma-\nu-1} \bar{B}_{\nu},
\end{gathered}
$$

where $k$ is a generally complex-valued variable, and

$$
\bar{A}_{\nu}=\tau_{n+\nu} A_{\nu}, \quad \bar{B}_{\nu}=\tau_{n+\nu} B_{\nu} .
$$

Thus, if $\bar{A}_{\nu}, \bar{B}_{\nu}$ can be chosen so that (25) holds, the functions $U_{n}, \psi_{n}, \phi_{n}{ }^{[h]}, \theta_{n}{ }^{[h]}$ will satisfy the difference equation whenever the series defining them converge, since (19)-(24) are all equivalent to (25)-(27).

We now discuss the quantity $\sigma$, which up till now has been unspecified.

Note that $f_{1}(k)$ is a polynomial in $k$ of degree $\sigma$ at most and, since no $b_{i}$ equals any $a_{i}$ or $\beta+1$, has zeros at $k=-n-a_{i}, i=1,2, \cdots, P+1$.

Or

$$
f_{1}(k) \equiv\left(n+a_{P+1}+k\right) M_{r}(k),
$$

where $M_{r}(k)$ is a polynomial of degree $r$ in $k$. Neither $f_{1}$ nor $M_{r}$ can be identically zero, since

$$
f_{1}(0)=(2 n+\gamma+1)_{\sigma} \bar{A}_{0} .
$$

Equation (29) shows that, for some integer $m_{1}, m_{1} \geqq 0, \sigma-m_{1}=P+r+1$ or $\sigma \geqq P+1$.

Likewise, $f_{2}$ is a polynomial of degree $\sigma-2$ at most and

$$
f_{2}(k)=\left(n+b_{Q}+k\right) N_{s}(k),
$$

where $N_{s}$ is a polynomial of degree $s$ in $k$. Setting $k=0$ in (25) gives

$$
\bar{B}_{1}=-\left(n+b_{Q}\right)(2 n+\gamma+1)_{2} \bar{A}_{0} /\left(n+a_{P+1}\right)
$$

and clearly this is the only possible value of $\bar{B}_{1}$. 
Furthermore,

$$
f_{2}(0)=-\left(n+b_{Q}\right)(2 n+\gamma+1)_{\sigma} \bar{A}_{0} /\left(n+a_{P+1}\right)
$$

so $N_{s}(k) \not \equiv 0, f_{2}(k) \not \equiv 0 ;(31)$ shows that, for some integer $m_{2} \geqq 0, \sigma-m_{2}-2=$ $Q+s$ or $\sigma \geqq Q+2$.

Thus, the smallest possible value of $\sigma$ is

$$
\sigma=\max [P+1, Q+2] .
$$

Assume $\sigma$ has this value. We will show that $\bar{A}_{\nu}, \bar{B}_{\nu}$ (hence, $A_{\nu}, B_{\nu}$ ) are then uniquely determined by (25) and that $A_{\sigma} \not \equiv 0$, which means that no other recursion relationship of order $\leqq \sigma$ exists for any of the given functions, i.e., statement (3) of the theorem. (It is clear, however, that larger values of $\sigma$ are possible, e.g., add to (12) the recursion relationship obtained by replacing $n$ by $n+1$ and the result is a recursion formula of order $\sigma+1$.)

LEMma 1. Let the conditions of the theorem hold. Then (25) is true if and only if $\bar{A}_{\nu}$, $\bar{B}_{\nu}$ are such that

$$
\begin{aligned}
& f_{1}(k) \equiv(2 n+\gamma+1)_{\sigma}\left(n+a_{P+1}+k\right) \bar{A}_{0} /\left(n+a_{P+1}\right), \\
& f_{2}(k) \equiv-(2 n+\gamma+1)_{\sigma}\left(n+b_{Q}+k\right) \bar{A}_{0} /\left(n+a_{P+1}\right) .
\end{aligned}
$$

If $k$ is assigned $\sigma$ distinct values in (35) and $\sigma-2$ distinct values in (36), then $\bar{A}_{\nu}$, $\nu=1,2, \cdots, \sigma$ and $\bar{B}_{\nu}, \nu=2,3, \cdots, \sigma-1$ are uniquely determined and so, by (28), are $A_{\nu}, B_{\nu}$. Also, $A_{\sigma} \not \equiv 0$.

Proof. First assume $P>Q+1, \sigma=P+1$. Then $f_{1}(k)$ is a polynomial of degree $P+1$ at most. But since $f_{1}(k) \not \equiv 0,(29)$ shows it must be exactly of degree $P+1$, and

$$
f_{1}(k)=K\left(n+a_{P+1}+k\right) \text {. }
$$

Letting $k=0$ and using (30) determines $K$, and when (35) is substituted into (25), (36) follows.

Let $P \leqq Q+1, \sigma=Q+2 ; f_{2}(k)$ is a polynomial in $k$ of degree $Q$ at most. As before, $f_{2}(k) \not \equiv 0$ and so

$$
f_{2}(k)=K^{\prime}\left(n+b_{Q}+k\right) .
$$

Letting $k=0$ and using (33) we find $K^{\prime}$ whence (36) follows. When (36) is substituted into (25), (35) results.

Now let $\sigma$ distinct values $k_{i}, i=1,2, \cdots, \sigma$ be assigned to $k$ in (35). The result is $\sigma$ nonhomogeneous equations in the $\sigma$ unknowns $\bar{A}_{\nu}, \nu=1,2, \cdots, \sigma$. Now this system has a unique solution which is independent of the values of $k$ assigned.

Let $V_{R}$ denote the alternate determinant

$$
V_{R}\left(x_{R}\right)=\left|x_{i}{ }^{j-1}\right|_{i, j=1,2, \cdots, R}=\prod_{m=2}^{R} \prod_{l=1}^{m-1}\left(x_{m}-x_{m-l}\right) .
$$

Here and in what follows, $\tau_{i j}$ is the element in the $i$ th row and $j$ th column of the determinant $\left|\tau_{i j}\right|_{i, j=1,2, \cdots, R}$. The determinant of the system formed from (35) is

$$
D=\mid(-)^{j-1}\left(1-k_{i}\right)_{j-1}\left(2 n+k_{i}+j+\gamma+1\right)_{\sigma-j \mid i, j=1,2, \cdots, \sigma}
$$

which, by [11], is 


$$
D=K V_{\sigma}\left(k_{\sigma}\right)
$$

and $K$ is independent of the $k_{i}$ 's. To determine $K$, let $k_{i}=i$. The resulting determinant is triangular, and we find

$$
D=V_{\sigma}\left(k_{\sigma}\right) \prod_{i=1}^{\sigma}(2 n+2 i+\gamma+1)_{\sigma-i}
$$

so, under our hypotheses, $D \neq 0$. If the system is solved by Cramer's rule, it can be verified that $V_{\sigma}\left(k_{\sigma}\right)$ also factors out of each numerator determinant, leaving a quantity independent of the $k_{i}$ 's. Thus, $\bar{A}_{\nu}$ is uniquely determined by (35), and similarly one can show that $\bar{B}_{\nu}$ is uniquely determined by (36), with $\bar{B}_{1}$ given by (32). $\bar{A}_{\sigma}$, hence $A_{\sigma}$, can be found by putting $k=-\sigma-\gamma-2 n$ in (35), and the result is displayed in Theorem 2, Eq. (52). Under our hypothesis, $A_{\sigma} \not \equiv 0$.

It remains to prove that $A_{\nu}, B_{\nu}$ are indeed given by (13) and (14). For this, we require two more lemmas.

Lemma 2. Let $k, b$ and $z$ be complex quantities, $b+k+1 \neq 0,-1,-2, \cdots$, and $s$ an integer $\geqq 0$. Then

$$
\sum_{\nu=0}^{8} \frac{(b+2 \nu)(-k)_{\nu}(b+z)_{\nu}}{(1-z)_{\nu}(b+k+1)_{\nu}}=\frac{z(k+b)+\frac{(-k)_{s+1}(b+z)_{s+1}}{(b+k+1)_{s}(1-z)_{s}}}{(z-k)} .
$$

Remark. Since the left-hand side and the right-hand side of (43) are the same meromorphic function of $z$, they have the same residues at the simple poles $z=1$, $2, \cdots, s$ and possess the same limit as $z \rightarrow k$.

Proof. By induction on $s$.

Lemma 3. If

$$
f_{k}=\sum_{\nu=0}^{k} \frac{(-k)_{\nu} g_{\nu}}{(a+k)_{\nu}}, \quad k=0,1,2, \cdots, M \geqq 0,
$$

then

$$
g_{\nu}=\frac{(a+2 \nu-1)}{\nu !} \sum_{s=0}^{\nu} \frac{(-\nu)_{s}(a+s)_{\nu} f_{s}}{s !(a+s+\nu-1)}
$$

provided $a \neq 0,-1,-2, \cdots$.

Proof. The determinant of the system is nonzero, so (44) has a unique solution. The lemma then results by substituting (45) in (44), interchanging the order of summation, and using Lemma 2 with $z=0$.

Now, in (35) let $k=0,1,2, \cdots, \sigma$. Then

$$
f_{k}=\sum_{\nu=0}^{k} \frac{(-k)_{\nu}(-)^{\nu} \bar{A}_{\nu}}{(2 n+\gamma+k+1)_{\nu}}=\frac{(2 n+\gamma+1)_{\sigma}\left(n+a_{P+1}+k\right) \bar{A}_{0}}{\left(n+a_{P+1}\right)(2 n+\gamma+k+1)_{\sigma}}
$$

and this system is the form in Lemma 3 with $g_{\nu}=(-)^{\nu} \bar{A}_{\nu}, a=2 n+\gamma+1$. Thus $\bar{A}_{\nu}$ and hence $A_{\nu}$ is easily found and the result is (13). $\bar{B}_{\nu}$ is similarly determined by applying Lemma 3 to (36).

The extension of the theorem to values $\lambda$ such that $|\arg (1-\lambda)|<\pi$ in Case A, $P=Q+1$, or $|\arg (1-1 / \lambda)|<\pi$ in Case $\mathrm{B}, P=Q+1$ is immediate by the permanence principle for functional equations [12]. 
The proof of Theorem 1 is complete.

Note that no restrictions on $b_{i}$ enter in the proof of the theorem; the restriction that $b_{i} \neq 0,-1,-2, \cdots$, arises from the definition (6). In fact, by slightly modifying (12) (e.g., multiplying by $\left(n+a_{P+1}\right)$ ) or the solutions of the difference equation (e.g., dividing $U_{n}(\lambda)$ by $\Gamma\left(b_{Q}\right)$ ), the theorem can be made valid for $a_{i}, b_{j}$ negative integers. Also, $\Phi_{n}$ may be redefined so that the theorem will hold for all values of $\beta+1$ and $\gamma$.

Now if no two of the quantities $\left[n, b_{Q},-\gamma-n\right]$ differ by an integer or zero, all the solutions in Case A are distinct, and if no two of the quantities $\left[a_{P+1}\right]$ differ by an integer or zero, all the solutions in Case B are distinct. In fact, under these restrictions the functions in each group are linearly independent functions of $\lambda$, as is seen by comparing their behavior near $\lambda=0$ or $\lambda=\infty$. This is not at all the same as asserting that the functions in either group are linearly independent as functions of $n$.

If $2 n+\gamma$ is an integer, $\psi_{n}(\lambda)$ is proportional to $U_{n}(\lambda)$, while if two of the quantities $\left[b_{Q}\right]$ (or $\left[a_{P+1}\right]$ ) differ by an integer or zero, then two of the functions $\left[\phi_{n}{ }^{[Q]}\right]$ (or $\left.\left[\theta_{n}{ }^{[P+1]}\right]\right)$ are proportional. However, in any of these cases a distinct set of solutions can be constructed. For example, let $a_{i}=a_{j}+m, m=0,1,2,3, \cdots$. Then one forms an appropriate difference of the functions $\theta_{n}{ }^{[i]}, \theta_{n}^{[i]}$ for $a_{i}=a_{j}+m+\epsilon$, divides by $\epsilon$, and lets $\epsilon \rightarrow 0$. See [13] for the mechanics of this procedure.

We will subsequently need the following integral representations of (13) and (14).

Lemma 4. Let none of the quantities $\gamma, a_{i}, i=1,2, \cdots, P+1$ be negative integers or zero. Then, for general $\sigma$, we have

$$
\begin{aligned}
A_{\nu} & =\frac{v_{n, \nu}}{2 \pi i} \int_{\Gamma_{\nu}} \frac{\Gamma(2 n+\gamma+\nu+z) \Gamma(-z)\left(n+a_{P+1}+z\right) d z}{\Gamma(2 n+\gamma+\sigma+1+z) \Gamma(\nu+1-z)}, \\
B_{\nu} & =\frac{v_{n, \nu}}{2 \pi i} \int_{\Gamma_{\nu-1}} \frac{\Gamma(2 n+\gamma+\nu+1+z) \Gamma(-z)\left(n+b_{Q}+z\right) d z}{\Gamma(2 n+\gamma+\sigma+1+z) \Gamma(\nu-z)}, \\
v_{n, \nu} & =\frac{(-)^{\nu+1}(2 n+\gamma)_{\sigma+1}(n+\beta+1)_{\nu}}{(n+\gamma)_{\nu}\left(a_{P+1}+n\right)}
\end{aligned}
$$

and $\Gamma_{m}$ denotes a simple closed path enclosing the points $z=0,1,2, \cdots, m$ but no other singularities of the integrand.

Proof. By the residue theorem. Note that $\Gamma_{m}$ is a feasible path since, were any of the poles of $\Gamma(2 n+\gamma+\nu+z)$ (or $\Gamma(2 n+\gamma+\nu+z+1))$ to coincide with any of the poles of $\Gamma(-z)$, then $\gamma$ would be zero or a negative integer.

We now give alternate representations of $A_{\nu}, B_{\nu}$ which are useful when $\nu$ is larger than $[\sigma / 2]$.

Theorem 2. Let none of the quantities $\gamma, \beta+1, a_{i}, i=1,2, \cdots, P$ be negative integers or zero. Then

$$
\begin{aligned}
& A_{\nu}=\frac{(-)^{\nu+P+1}(2 n+\gamma)_{\sigma+1}(n+\beta+1)_{\nu}\left(n+\gamma+\nu-a_{P+1}\right)}{\Gamma(\sigma+1-\nu)(n+\gamma)_{\nu}(2 n+\gamma+\nu)_{\nu+1}\left(n+a_{P+1}\right)} \\
& \times_{P+3} F_{P+2}\left(\begin{array}{l|l}
\nu-\sigma, 2 n+\gamma+\nu, n+\gamma+\nu+1-a_{P+1} & 1 \\
2 n+\gamma+2 \nu+1, n+\gamma+\nu-a_{P+1} & 1
\end{array}\right),
\end{aligned}
$$




$$
\begin{aligned}
B_{\nu}= & \frac{(-)^{\nu+Q}(2 n+\gamma)_{\sigma+1}(n+\beta+1)_{\nu}\left(n+\gamma+\nu+1-b_{Q}\right)}{\Gamma(\sigma-\nu)(n+\gamma)_{\nu}(2 n+\gamma+\nu+1)_{\nu}\left(n+a_{P+1}\right)} \\
& \quad \times{ }_{Q+2} F_{Q+1}\left(\begin{array}{l}
\nu+1-\sigma, 2 n+\gamma+\nu+1, n+\gamma+\nu+2-b_{Q} \\
2 n+\gamma+2 \nu+1, n+\gamma+\nu+1-b_{Q}
\end{array}\right.
\end{aligned}
$$

and in particular

$$
A_{\sigma}=\frac{(-)^{\sigma+P+1}(2 n+\gamma)_{\sigma}(n+\beta+1)_{\sigma}\left(n+\gamma+\sigma-a_{P+1}\right)}{(n+\gamma)_{\sigma}(2 n+\gamma+\sigma+1)_{\sigma}\left(n+a_{P+1}\right)} .
$$

Proof. We prove (50) only, since (51) follows similarly. Denote the integrand of (47) by $L_{n}(z)$. It has poles at the points $\delta_{m}=-2 n-\gamma-m, m=\nu, \nu+1, \cdots, \sigma$ and $\gamma_{m}, m=0,1,2, \cdots, \nu$. The integral around any large circle containing both $\left\{\gamma_{m}\right\}$ and $\left\{\delta_{m}\right\}$ is zero, since $L_{n}(z)=O\left\{z^{P-\sigma-1}\right\},|z| \rightarrow \infty$, and is a rational function of $z$. If $\Delta_{\nu}$ is any simple closed curve containing the points $\left\{\gamma_{m}\right\}$ but none of the points $\left\{\delta_{m}\right\}$, then

$$
\int_{\Gamma_{\nu}}=-\int_{\Delta_{\nu}}
$$

and (50), and hence (52), follow immediately by the residue theorem. (Note the hypotheses separate the points $\left\{\gamma_{m}\right\}$ from $\left\{\delta_{m}\right\}$.)

Because of the form of the functions $\theta_{n}{ }^{[h]}(\lambda)$, Theorems 1 and 2 enable us to give explicit recurrence formulae for the classes of hypergeometric polynomials studied in [4].

Corollary 1. Let $R$ and $T$ be integers $\geqq 0, \tau=\max [T+1, R+2]$. Let $\gamma, c_{i}$, $d_{j}, i=1,2, \cdots, R, j=1,2, \cdots, T+1,\left(d_{j}=1\right.$ for $\left.j=T+1\right)$ be complex constants such that none of the quantities $\gamma, \gamma+1-d_{j}, j=1,2, \cdots, T$ are negative integers or zero. Then the hypergeometric polynomials $P_{n}(z)$, see (7), satisfy the recursion relationship

$$
\sum_{\nu=0}^{\tau}\left[C_{\nu}+z D_{\nu}\right] P_{n-\nu}(z)=0, \quad n=\tau, \tau+1, \tau+2, \cdots,
$$

where

$$
\begin{aligned}
C_{\nu}= & \frac{(-)^{\nu}(n+1-\nu)_{\nu}(1-\gamma-2 n)_{2 \nu}\left(n-\nu-1+d_{T+1}\right)}{\nu !(n+\gamma-\nu)_{\nu}(\tau+1-\gamma-2 n)_{\nu}\left(n+d_{T+1}-1\right)} \\
& \times{ }_{{ }_{T+3}} F_{T+2}\left(\begin{array}{c|c}
-\nu, 2 n+\gamma-\tau-\nu, n-\nu+d_{T+1} & 1 \\
2 n+\gamma+1-2 \nu, n-\nu-1+d_{T+1} & 1
\end{array}\right)
\end{aligned}
$$

and

$$
\begin{aligned}
D_{\nu}= & \frac{(-)^{\nu+1}(n+1-\nu)_{\nu}(1-\gamma-2 n)_{2 \nu}\left(n-\nu+c_{R}\right)}{\Gamma(\nu)(n+\gamma-\nu)_{\nu}(1+\tau-\gamma-2 n)_{\nu-1}\left(n+d_{T+1}-1\right)} \\
& \times_{R+2} F_{R+1}\left(\begin{array}{l|l}
1-\nu, 2 n+\gamma+1-\tau-\nu, n+1-\nu+c_{R} & 1 \\
2 n+\gamma+1-2 \nu, n-\nu+c_{R}
\end{array}\right.
\end{aligned}
$$

and $D_{0}=D_{\tau}=0$.

Proof. In $\theta_{n}{ }^{[P+1]}(\lambda)$ let $Q=R, P=T, a_{j}=\gamma+1-d_{j}\left(d_{T+1}=1\right), b_{j}=\gamma+$ $1-c_{j}, \beta+1=\gamma, z=(-)^{Q+P+1} / \lambda, \sigma=\tau$. Then (55) and (56) follow from Theo- 
rem 2 when the sums are turned around and $n$ is replaced by $n-\tau$; since the polynomials are computed in the forward direction, this is the more useful form of the recursion relationship. Note that it is not necessary to assume $P>Q+1$ in using Theorem 2. Since $\theta_{n}^{[P+1]}(\lambda)$ terminates, the recursion formula is valid for all $P, Q$. Also, alternate forms for $C_{\nu}, D_{\nu}$ which are useful when $\nu>[\sigma / 2]$ can be determined from Theorem 1.

Corollary 2. Let $R$ and $T$ be integers $\geqq 0, \tau=\max [T+1, R+2]$, and let $c_{i}$, $d_{j}, i=1,2, \cdots, R, j=1,2, \cdots, T+1$ be complex constants, $\left(d_{j}=1\right.$ for $j=T$ $+1)$. Then the hypergeometric polynomials $Q_{n}(z)$, see (8), satisfy the recursion relationship

$$
\sum_{\nu=0}^{l_{1}} E_{\nu} Q_{n-\nu}(z)+z \sum_{\nu=1}^{l_{2}} F_{\nu} Q_{n-\nu}(z)=0,
$$

$l_{1}=\min [\tau, T+1], l_{2}=\min [\tau-1, R+1], n=\tau+\delta, \tau+\delta+1, \tau+\delta+$ $2, \cdots, \delta=0$ or -1 , where

$$
\begin{aligned}
& E_{\nu}=\frac{(n+1-\nu)_{\nu}\left(n-\nu-1+d_{T+1}\right)}{\nu !\left(n+d_{T+1}-1\right)}{ }_{T+2} F_{T+1}\left(\begin{array}{c}
-\nu, n-\nu+d_{T+1} \\
n-\nu-1+d_{T+1}
\end{array} \mid 1\right), \\
& F_{\nu}=\frac{(n+1-\nu)_{\nu}\left(n-\nu+c_{R}\right)}{\Gamma(\nu)\left(n+d_{T+1}-1\right)}{ }_{R+1} F_{R}\left(\begin{array}{c}
1-\nu, n+1-\nu+c_{R} \\
n-\nu+c_{R}
\end{array}\right) .
\end{aligned}
$$

Proof. Let

$$
Q_{n}{ }^{(\gamma)}(z)=P_{n}(z / \gamma)
$$

Then

$$
\lim _{\gamma \rightarrow \infty} Q_{n}^{(\gamma)}(z)=Q_{n}(z) .
$$

If we form the difference equation for $Q_{n}{ }^{(\gamma)}(z)$ we see we must have

$$
\lim _{\gamma \rightarrow \infty} C_{\nu}=E_{\nu}, \quad \lim _{\gamma \rightarrow \infty} \gamma^{-1} D_{\nu}=E_{\nu} .
$$

Using (55), (56) to take the limits term by term gives (58) and (59).

Note that $E_{\nu}$ vanishes for $\nu>T+1$ and $F_{\nu}$ for $\nu>R+1$ since they may be expressed as the $\nu$ th difference of $\left(n+d_{T+1}-1-\nu+x\right)$ or the $(\nu-1)$ th difference of $\left(n+c_{R}-\nu+x\right)$ respectively evaluated at $x=0$.

Midwest Research Institute

Kansas City, Missouri

1. A. Erdélyi, W. Magnus, F. Oberhettinger \& F. G. Tricomi, Higher Transcendental Functions, Vol. 3, McGraw-Hill, New York, 1955; Vol. 1, 1953, Chapters 4, 5. MR 15, 419; MR 16, 586.

2. JET WIMP \& Y. L. LUKE, "Expansion formulas for generalized hypergeometric functions," Rend. Circ. Mat. Palermo (2), v. 11, 1962, pp. 351-366. MR 29 \#3681.

3. Y. L. LUKE \& JET WIMP, "Jacobi polynomial expansions of a generalized hypergeometric function over a semi-infinite ray," Math. Comp., v. 17, 1963, pp. 395-404. MR 28 \#255.

4. J. L. FIELDS \& JET WIMP, "Expansions of hypergeometric functions in hypergeometric functions," Math. Comp., v. 15, 1961, pp. 390-395. MR 23 \#A3289.

5. A. Erdélyi, W. Magnus, F. Oberhettinger \& F. G. Tricomi, Higher Transcendental Functions, Vol. 2, McGraw-Hill, New York, 1953, Chapter 10. MR 15, 419. 
6. J. L. FIELDS \& JET WIMP, "Basic series corresponding to a class of hypergeometric polynomials," Proc. Cambridge Philos. Soc., v. 59, 1963, pp. 599-605. MR 27 \#351.

7. Y. L. LukE, “On economic representations of transcendental functions," J. Math. Phys., v. 38, 1960, pp. 279-294. MR 22 \#3829.

8. JET Wimp, "On the zeros of a confluent hypergeometric function," Proc. Amer. Math. Soc., v. 16, 1965, pp. 281-283. (Also the references given there.) MR 30, \#4001.

9. E. D. Rainville, Special Functions, Macmillan, New York, 1960, p. 233 ff. MR 21 \#6447.

10. Reference 1, Vol. 1, p. 208, (5)-(6).

11. Thomas Muir, $A$ Treatise on the Theory of Determinants, Dover, New York, 1960, Chapter 9. MR 22 \#5644.

12. ZeEv Nehari, Conformal Mapping, McGraw-Hill, New York, 1952, p. 107. MR 13, 640.

13. Y. L. LukE, Integrals of Bessel Functions, McGraw-Hill, New York, 1962, p. 14. MR 25 \#5198. 